\title{
The efficacy of laparoscopic lauromacrogol sclerotherapy in the treatment of simple hepatic cysts located in posterior segments: a refined surgical approach
}

\author{
Song $\mathrm{Xu}^{1}$, Min $\mathrm{Rao}^{1}$, Yong $\mathrm{Pu}^{2}$, Junjing Zhou ${ }^{1}$, Yong Zhang ${ }^{1}$ \\ ${ }^{1}$ Department of Hepatobiliary Surgery, Affiliated Hospital of Jiangnan University, Wuxi, China; ${ }^{2}$ Department of Pathology, Affiliated Hospital of \\ Jiangnan University, Wuxi, China \\ Contributions: (I) Conception and design: S Xu; (II) Administrative support: Y Pu; (III) Provision of study materials or patients: Y Zhang; (IV) \\ Collection and assembly of data: M Rao; (V) Data analysis and interpretation: J Zhou; (VI) Manuscript writing: All authors; (VII) Final approval of \\ manuscript: All authors. \\ Correspondence to: Yong Zhang. Department of Hepatobiliary Surgery. The Affiliated Hospital of Jiangnan University, 200 Huihe Road, Wuxi, China. \\ Email: spectrum163@163.com.
}

Background: Hepatic cysts in located posterior segments close to the diaphragm (IVa, VII, and VIII) reportedly have a high recurrence rate. Presently, laparoscopic omentoplasty is the accepted technique; developed from laparoscopic deroofing, which places a viable pedicle flap of omentum to prevent cyst closure. However, potential adhesions have made laparoscopic omentoplasty less favorable. In this paper, we report on an improved surgical technique involving lauromacrogol sclerosis directly under laparoscopic fenestration. We also review and evaluate the efficacy and feasibility of this refined surgical approach.

Methods: Data from 49 patients admitted to the Department of Hepatobiliary Surgery at the Affiliated Hospital of Jiangnan University from October 2015 to June 2020 with simple hepatic cysts located in the IVa, VII, and VIII segments were retrospectively analyzed. All patients were symptomatic before admission. They were separated into two groups based on the surgical approach they had received; refined laparoscopic lauromacrogol sclerotherapy or laparoscopic omentoplasty, and were compared and evaluated in terms of the postoperative cyst volume and quality of life.

Results: No significant differences in sex, age, preoperative cyst volume, surgery duration, hospital stay, and bleeding volume were reported. There were no deaths or major complications in both groups. The postoperative cyst volume was significantly reduced in the laparoscopic lauromacrogol sclerotherapy group $(2.48 \mathrm{~cm})$ compared to the laparoscopic omentoplasty group $(3.90 \mathrm{~cm})$. This study evaluated both the immediate and medium-term results with a 3-12 months follow-up period for all patients. The cyst volume change in the laparoscopic lauromacrogol sclerotherapy group was found to be significantly greater than that of the laparoscopic omentoplasty group. The feedback regarding quality of life did not vary significantly between the two groups, except for general health and health change, where patients who received laparoscopic sclerotherapy responded with higher scores.

Conclusions: Our results indicate that laparoscopic lauromacrogol sclerotherapy surgery was safe and effective in patients with IVa, VII and VIII segment simple hepatic cysts.

Keywords: Laparoscopic lauromacrogol sclerotherapy surgery; laparoscopic omentoplasty; posterior segments cysts; efficacy; quality of life

Submitted Aug 07, 2020. Accepted for publication Sep 14, 2020.

doi: 10.21037/apm-20-1723

View this article at: http://dx.doi.org/10.21037/apm-20-1723 


\section{Introduction}

Hepatic cysts are a common benign liver disease with a prevalence of $5-10 \%$ (1). Small cysts typically cause no or minor symptoms such as nausea, vomiting, and upper abdominal pain. However, when cysts increase in size and volume, patients can develop acute abdominal symptoms and complications including hemorrhage, rupture, infection, and torsion (2-5). Clinical intervention usually occurs in cases that are symptomatic, and various approaches have been advocated. Surgical procedures such as laparoscopic fenestration, laparoscopic omentoplasty and resection and non-surgical procedure, for example, percutaneous aspiration have been applied to treat symptomatic cysts $(6,7)$. Sclerotherapy is one such procedure, and is performed by injecting sclerosing agents into cysts. However, there remains considerable controversy among surgeons regarding the most appropriate surgical approach (8). In fact, it has been argued that the application of the various approaches in consideration of their indication and complications, depends on the size, type, and position of cysts in the liver (9). In particular, this paper focuses on the treatment of simple hepatic cysts located in difficult segments (IVa, VII, and VIII).

Laparoscopic deroofing is a widely accepted treatment option for simple hepatic cysts. However, some studies have reported a high recurrence rate following laparoscopic fenestration to deroof cysts located in the diaphragmatic surface, typically in difficult segments (IVa, VII, and VIII) $(10,11)$. This could be due to the tendency of the diaphragm to close the roof. In response, laparoscopic omentoplasty was proposed to reduce the potential risk of roof closure (12). The surgery approach is considered to be effective in preventing postoperative complications (13). Meanwhile, treatment with puncture and sclerosis under ultrasound guidance was also introduced. The undeniable advantages of this safe, effective, and minimally invasive treatment have led this technique becoming increasingly popular (14). It has, however, been associated with pain and potential ethanol intoxication (15). Moreover, the reported recurrence rate is much higher than laparoscopic deroofing and laparoscopic omentoplasty (16). Apart from the above methods, direct cyst excision has also been proposed for the treatment of symptomatic cysts. Despite zero recurrence rate, however, it is associated with higher morbidity rate. Put simply, there is presently no ideal treatment approach. In fact, hepatic surgery has been criticized for the lack of demonstrated benefits in terms of the quality of current surgical procedures (17).

To reduce recurrence rate, prevent potential adhesions caused by omentum, and perform treatment on patients without enough omentum flap, we propose a refined surgical technique that applies lauromacrogol sclerosis under laparoscopic fenestration. The purpose of this study is to report on the laparoscopic lauromacrogol sclerotherapy surgical technique in the treatment of simple hepatic cysts. We retrospectively studied 49 patients admitted to the Department of Hepatobiliary Surgery of the Affiliated Hospital of Jiangnan University between October 2015 and June 2020 with simple hepatic cysts located in IVa, VII, and VIII segments. In these cases, either laparoscopic omentoplasty or the refined laparoscopic lauromacrogol sclerotherapy surgery was performed. We compared and evaluated the efficacy of the laparoscopic lauromacrogol sclerotherapy surgical technique in terms of recurrence, postoperative cyst volume change, and most importantly, postoperative quality of life. We present the following article in accordance with the STROBE reporting checklist (available at http://dx.doi.org/10.21037/apm-20-1723).

\section{Methods}

\section{Study design}

A total of 57 patients admitted to the Department of Hepatobiliary Surgery of the Affiliated Hospital of Jiangnan University between October 2015 and June 2020 and diagnosed with IVa, VII, and VIII liver cysts by ultrasonography or computed tomography (CT) scan were initially included in our study. Patients reported symptoms including abdominal compression, abdominal distension, or dull pain prior to hospital admission. Eight patients were excluded because of missing information, leaving 49 patients available for analysis (including 16 males and 33 females; median age, 60 years; age range, $45-78$ years). Two surgical techniques were employed: laparoscopic lauromacrogol sclerotherapy and laparoscopic omentoplasty, and patients were separated into two groups based on which of the aforementioned surgical techniques they had received. We retrospectively compared the efficacy of both surgical approaches in terms of hospital stay, recurrence rate, change in postoperative cyst diameter, and quality of life. Patients were invited to visit every 3-6 months, and a clinical examination, ultrasonography or CT scan, and a detailed questionnaire assessing health and quality of life were performed. The longest follow-up period was 12 months 

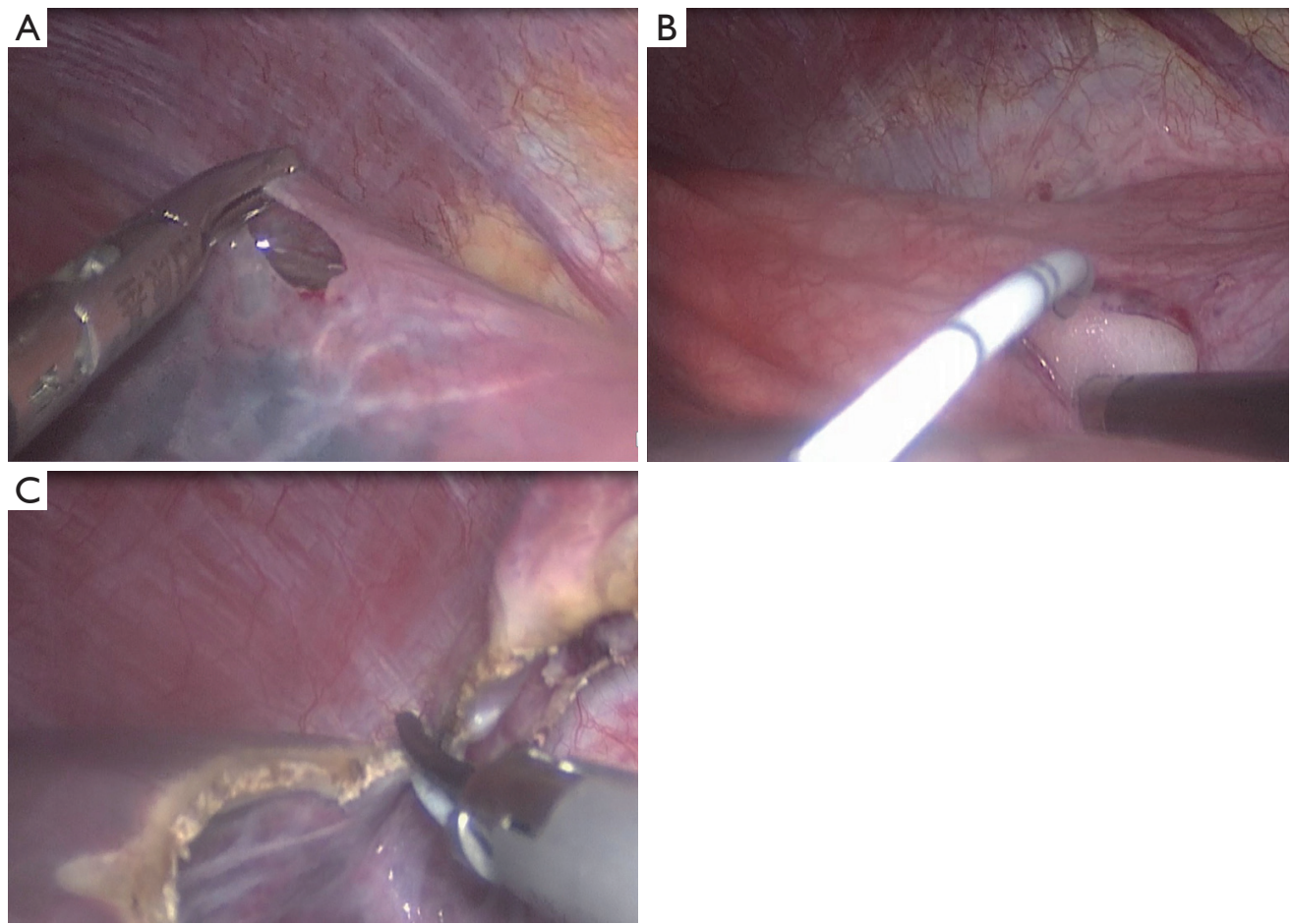

Figure 1 Refined surgical approach of laparoscopic lauromacrogol sclerotherapy. (A) A small incision at the tip of the cyst. (B) The lauromacrogol injection. (C) Deroofing using a harmonic scalpel after sclerotherapy.

after surgery. Specifically, we utilized the Short-Form (SF)36 questionnaire developed by RAND Health (https://www. rand.org/health-care/surveys_tools/mos/36-item-shortform/survey-instrument.html; RAND Corp., Cambridge, UK), which employs a composite scoring system ranging from 0 to 3,600 to indicate quality of life, with a higher score indicating a more favorable health state (RAND 36Item Health Survey 1.0). This survey taps health concepts including physical functioning, role limitations due to physical health and personal problems, emotional wellbeing, social functioning, energy, and general health. The study was conducted in accordance with the Declaration of Helsinki (as revised in 2013). The study was approved by the Medical Ethics Committee of the Affiliated Hospital of Jiangnan University (No. LS2015041) and written informed consent was obtained from all patients.

\section{Surgical techniques}

All surgeries were performed under general anesthesia. Patients were usually placed in the supine position. A $10 \mathrm{~mm}$ trocar was inserted through an umbilical incision and pneumoperitoneum was established. A $12 \mathrm{~mm}$ trocar was inserted below the xiphoid and a $5 \mathrm{~mm}$ trocar was inserted at the right mid clavicular line After the cyst was located, it was punctured using a laparoscopic needle. If the cyst was located at the VII segment, the patient was placed in the left oblique position. All laparoscopic punctures were then moved $10 \mathrm{~mm}$ to the right correspondingly. Next, expose the liver after sectioning the ligaments with the harmonic scalpel.

For the laparoscopic lauromacrogol sclerotherapy group (Group A), we began with a $5-10 \mathrm{~mm}$ incision at the tip of the cyst using a harmonic scalpel (see Figure 1). The intracystic fluid was inspected (clear, brownish, or limpid) and aspirated. A ureteral catheter was then placed into the incision and injected with the prepared sclerosing agent (1\% lauromacrogol, Tianyu Chang'an Group, Xi'an city, China). To create the foam, the Tessari method was employed. A three-way connecting pipe was used with two $20 \mathrm{~mL}$ injectors: one with the lauromacrogol injection and the other with air. The air and lauromacrogol were injected together at a gas to liquid ratio of 2:1. The injection amount was a quarter of the capsule fluid, and the liquid was pumped with air repeatedly several times until an entirely foamy consistency was achieved. The lauromacrogol 
Table 1 Characteristics of patients

\begin{tabular}{|c|c|c|c|c|}
\hline Variable & All patients (mean $\pm \mathrm{SD}$ ) & Group A (mean \pm SD) & Group B (mean \pm SD) & $P$ value \\
\hline Male, n (\%) & $16(32.6 \%)$ & 9 & 7 & 0.49 \\
\hline Age (years) & $62.06 \pm 9.11$ & $61.83 \pm 9.12$ & $62.28 \pm 9.29$ & 0.87 \\
\hline Cyst diameter (before) & $11.94 \pm 1.36$ & $12.19 \pm 1.50$ & $11.71 \pm 1.19$ & 0.22 \\
\hline Cyst diameter change $^{\star \star \star}$ & $74.70 \%$ & $80.77 \%$ & $68.87 \%$ & 0.001 \\
\hline Surgery duration (min) & $71.02 \pm 35.18$ & $70.21 \pm 36.79$ & $71.8 \pm 34.31$ & 0.88 \\
\hline Bleeding volume (mL) & $33.57 \pm 25.86$ & $29.17 \pm 27.25$ & $37.8 \pm 24.24$ & 0.25 \\
\hline Drainage duration (days) & $3.10 \pm 1.73$ & $2.88 \pm 1.62$ & $3.32 \pm 1.84$ & 0.38 \\
\hline
\end{tabular}

${ }^{* \star \star}$, statistically significant at $1 \%$ level.

injection was in full contact with the cystic space and lauromacrogol was supplemented when necessary. After 15 minutes, the lauromacrogol was sucked out and washed with saline. Finally, deroofing was performed using a harmonic scalpel.

For the laparoscopic omentoplasty group (Group B), the surgical procedure was generally performed according to the principles described by Lin et al. (18). The cyst was unroofed using a harmonic scalpel. The cyst cavity was then filled with the omentum flap with vessel pedicle of $10-15 \mathrm{~cm}$ in length. The upper and middle section of the omentum flap was then sutured and fixed to the cyst wall using an absorbable suture, leaving the top section of the omentum flap at the bottom of the cyst. The cystic walls were sampled for routine pathological examination and a drain was left in place. Three patients in the laparoscopic omentoplasty group with cholecystolithiasis received cholecystectomy simultaneously.

\section{Statistical analysis}

Data were obtained and collected from manual and electronic patient file. The simple hepatic cysts are nonPCLD cysts. In particular, this study focuses on simple hepatic cysts located in IVa, VII, and VIII segments. In particular, data were analyzed using Stata software. Measurement data are presented as mean \pm standard deviation. Since the sample size was relatively small, the student $t$-test and the Chi-square $\left(\chi^{2}\right)$ test were applied for null hypotheses. For related samples the paired $t$-test were used. The postoperative SF-36 scores were compared using a paired $t$-test and presented in mean and standard deviation. This research attempted to achieve the maximum follow-up rate to reduce potential bias. The final follow-up rate is $85 \%$.

\section{Results}

A total of 49 patients including 33 females and 16 males who received laparoscopic surgery were identified. There were no incidences of hemorrhage or biliary injury during the operations. The postoperative pathological examination found that all patients had simple hepatic cysts, and the CT scan results showed that fenestration was achieved for all patients. There were no severe postoperative complications such as bleeding, bile leakage, infection, and hepatic injury. 2 patients that received laparoscopic omentoplasy treatment reported to have minor gastrointestinal dysfunction. The characteristics and general descriptive statistics are shown in Table 1.

Group A includes the data of 24 patients who received laparoscopic lauromacrogol sclerotherapy, which involved laparoscopic fenestration with lauromacrogol sclerosis. Figure 2 shows clear necrosis of the cyst wall, suggesting the success of the refined surgical approach. One case in this group reported pain in the lower right back following the operation, although this pain disappeared the next day after treatment with a Tramadol injection. Group B includes the sample results of 25 patients who received laparoscopic omentoplasty. There were no significant differences 

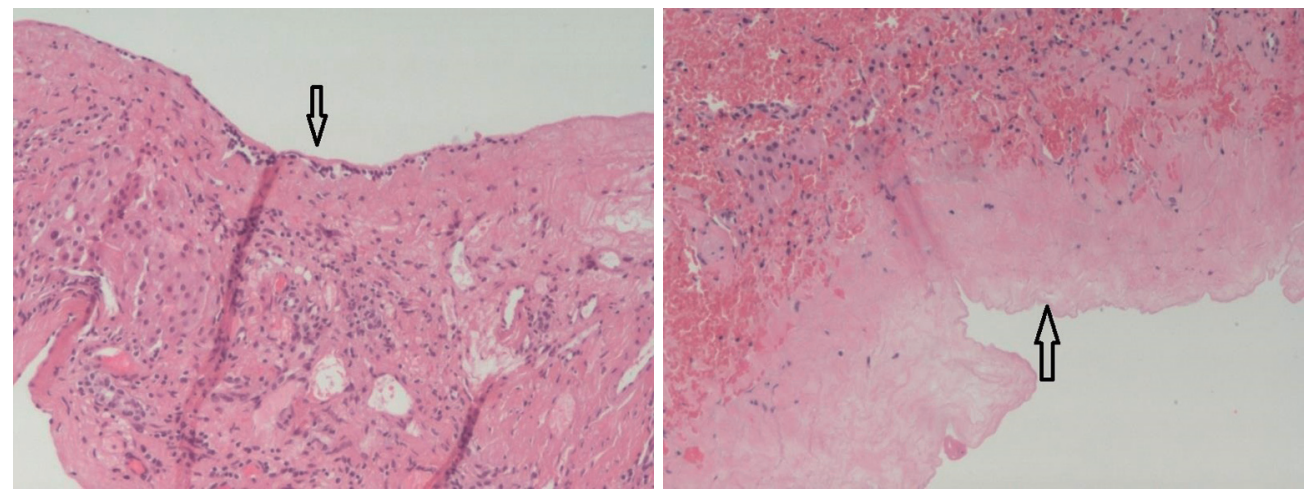

Figure 2 Cyst wall $(\times 200)$ (left) and cyst wall after lauromacrogol sclerosis $(\times 200)$ (right).

Table 2 The efficacy of surgery techniques: cyst volume change

\begin{tabular}{lccccc}
\hline Variable & Curative & Significant & Effective & Ineffective & Efficiency \\
\hline Group A & 5 & 13 & 5 & $15.83 \%$ & $92 \%$ \\
Group B & 0 & 8 & & & \\
$\chi^{2}$ & & 11.5082 & & & 0.009 \\
$P$ value & & & & 95 \\
\hline
\end{tabular}

between Group A and Group B patients in terms of sex, age, surgery duration, bleeding volume during the operation, drainage duration, and hospital stay $(\mathrm{P}>0.05)$. However, the mean cyst diameters were had decreased significantly in Group A (80.77\% reduction) compared to Group B (68.87\% reduction) $(\mathrm{P}=0.001)$. Table 2 describes the detailed postoperative cyst volume statistics of the two groups, with four aspects being identified to evaluate the efficacy of surgery results. The average follow-up period is 4 months after surgery. In this study, patients were considered to be cured if the cyst disappeared completely or was $90 \%$ smaller than its preoperative size. Patients with cysts that were $75-$ $90 \%$ smaller than their preoperative size were considered to be at a significant treatment level, and those with cysts that were $50-75 \%$ smaller in size were classified into the effective treatment level. We defined cyst changes of less than $50 \%$ as ineffective. In general, both surgical techniques were effective in reducing cyst volume. The detailed frequency table shows that among patients who received laparoscopic lauromacrogol sclerotherapy, there were five patients whose cysts almost completely disappeared, whereas none of the patients who received laparoscopic omentoplasty reported such change. Apart from curative results, more patients in Group A were classified in the significant level (75-90\% reduction in cyst size) compared to Group B (Group A =13, Group B =8). Figure 3 shows the pre-and post-operative CT scan results for both groups. It is important to note that there were no cases of recurrence among all patients in both groups.

With regards to quality of life, there were no statistical differences in the overall scores between the two groups $(\mathrm{P}=0.29)$. Table 3 and Figure 4 show the statistical analysis of the postoperative quality of life scores. The quality of life score refers specifically to physical functioning, body pain, role limitations due to physical and personal problems, emotional well-being, social functioning, energy, general health perceptions, and health change. In general, quality of life did not vary significantly among patients in both groups. Although, the general health status $(\mathrm{P}=0.04)$ and health change (compared to 1 year ago) $(\mathrm{P}=0.06)$ is slightly higher for patients who received laparoscopic lauromacrogol sclerotherapy.

\section{Discussion}

A number of previous studies have addressed the treatment of simple hepatic cysts. There is considerable controversy among surgeons with regards to the most appropriate 

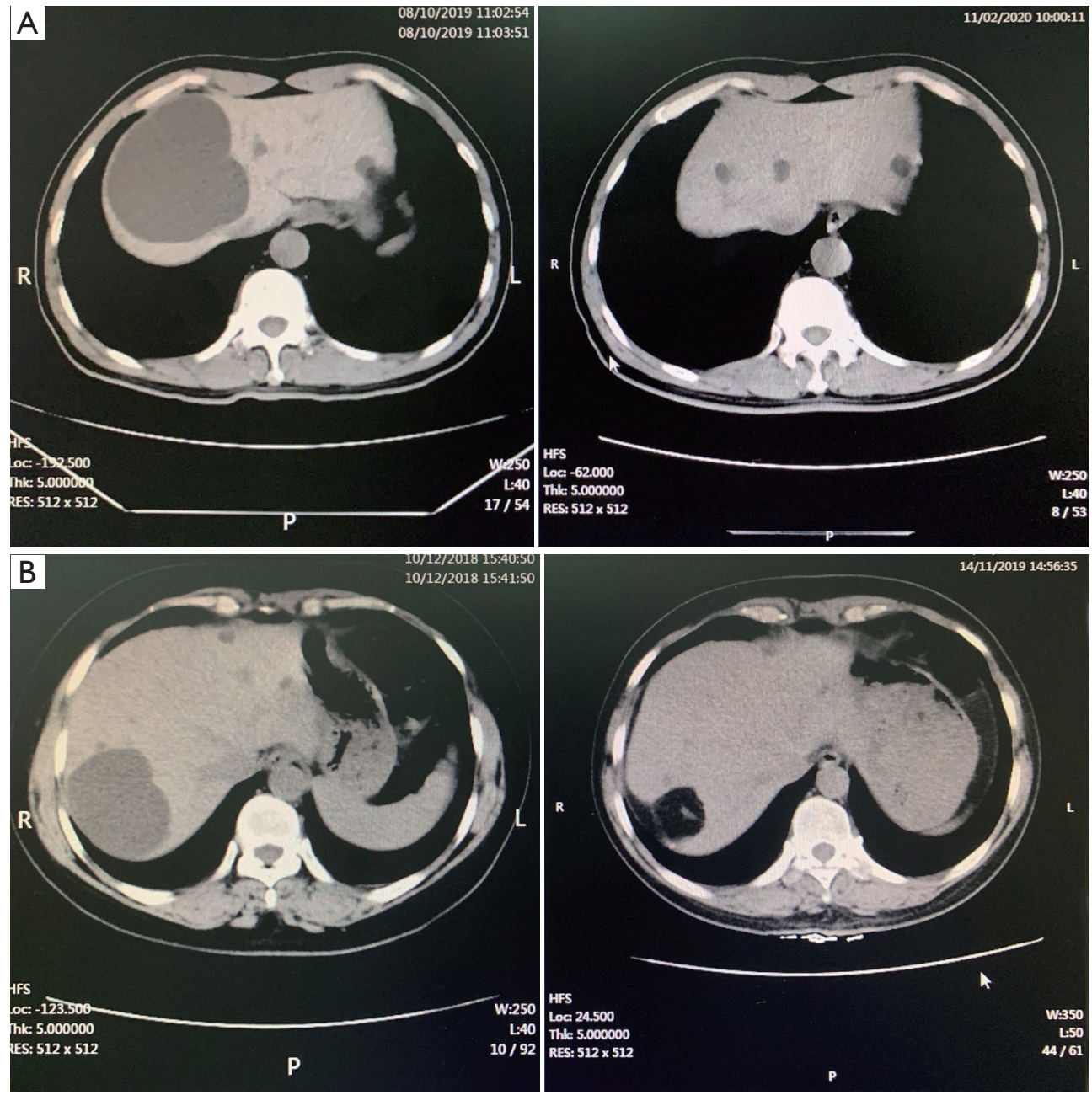

Figure 3 Preoperative (left) and postoperative (right) CT scan for Group A (up) and Group B (down).

surgical techniques, especially in terms of recurrence and morbidity rates. Radical routines, such as cyst excision, have been proposed for the treatment of symptomatic cysts $(19,20)$, with a reported recurrence rate of zero. However, such approaches are often associated with higher morbidity, and are therefore not the primary treatment option for patients, especially those with benign disease. In fact, this is the primary reason that laparoscopic technology has become well developed and is the choice of treatment in hepatic surgery. With safe and less invasive procedures, patients benefit immediately from smaller laparotomy incisions.

Laparoscopic deroofing is considered an effective and feasible surgical approach $(21,22)$, and has been recognized as safe especially for cysts located in segments II, III, IV, V, and VI of the hepatic lobe (16). However, recurrences have been consistently reported in the literature, with rates of up to $15 \%$ according to Maruyama et al. [2013] (23). Thus, it is critical that factors leading to clinical recurrence are identified. Previous studies have indicated that inadequate/ insufficient deroofing or laparoscopic deroofing can lead to adhesion formation, which also encourages cyst recurrence (24). Moreover, some deep-seated cysts and those located in close proximity to the diaphragm have been found to increase the likelihood of recurrence (25).

Surgical outcomes are significantly related to the location of cysts (26), especially for cysts in the VII, VIII, and IVa segments. There are technical clinical difficulties in performing a complete laparoscopic deroofing, while adhesions can often be found between the cyst and the diaphragm. As for the technical difficulty, Weber et al. [2004] introduced a special position of laparoscopic fenestration for successful treatment of VII and VIII cysts (27). Yet, 
Table 3 Statistic analysis of quality of life scores

\begin{tabular}{|c|c|c|c|c|}
\hline Variable & All patients (mean $\pm \mathrm{SD}$ ) & Group A (mean \pm SD) & Group B (mean \pm SD) & $P$ \\
\hline Physical functioning & $874.49 \pm 48.00$ & $881.25 \pm 50.67$ & $868 \pm 45.37$ & 0.34 \\
\hline Bodily pain & $177.35 \pm 22.92$ & $180.42 \pm 25.49$ & $174.4 \pm 20.22$ & 0.36 \\
\hline Physical limitation & $251.02 \pm 50.51$ & $250 \pm 51.08$ & $252 \pm 50.99$ & 0.89 \\
\hline Emotional well-being & $349.80 \pm 48.15$ & $353.33 \pm 44.00$ & $346.4 \pm 52.51$ & 0.62 \\
\hline Social functioning & $174.49 \pm 26.26$ & $178.13 \pm 25.87$ & $171 \pm 26.69$ & 0.35 \\
\hline Energy & $289.39 \pm 36.31$ & $288.33 \pm 31.16$ & $290.4 \pm 41.28$ & 0.84 \\
\hline General health** & $296.94 \pm 56.51$ & $313.54 \pm 61.67$ & $281 \pm 46.93$ & 0.04 \\
\hline
\end{tabular}

${ }^{*}$, statistically significant at $10 \%$ level; **, statistically significant at $5 \%$ level.

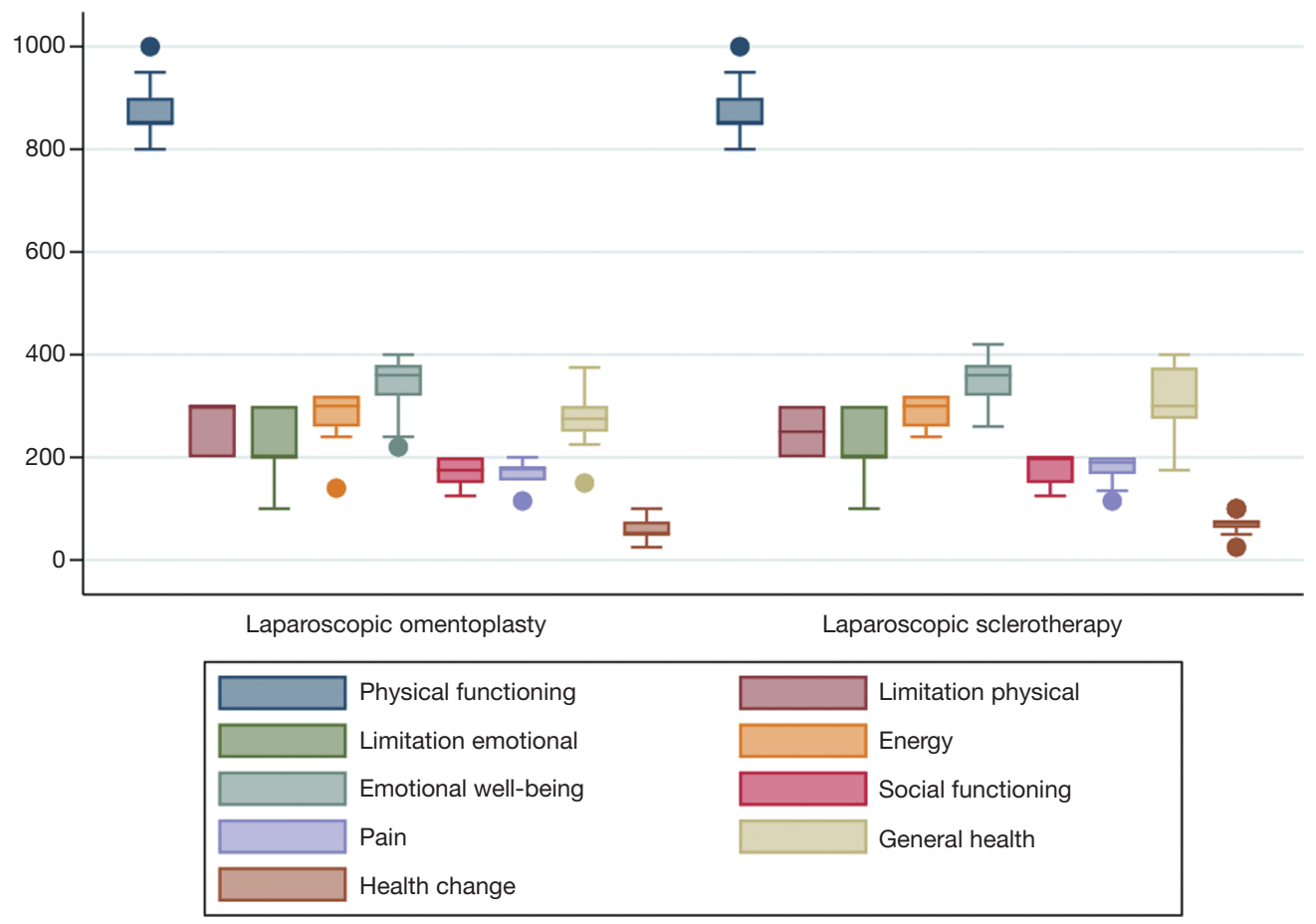

Figure 4 Efficacy of both surgical techniques in terms of quality of life scores.

recurrence still exists, as the cyst tends to close, and the epithelial lining continues to secrete serous fluid that accumulates in the cyst. So, the outcome of laparoscopic deroofing for hepatic cysts located in IVa, VII, and VIII segments is inferior to those located in other segments.

Correspondingly, the transposition of the omentum to the cyst bed has been advocated to reduce the risk of cyst recurrences (28). In our study, the Group B results supported the efficacy of this method in terms of the postoperative cyst volume and quality of life. However, some patients that do not present enough omentum flap to cover the extensively fenestrated hepatic surface are 
not eligible for this surgical approach (29). Meanwhile, 2 out of 25 patients reported to have minor gastrointestinal dysfunction. This might be because that omentum flap has a drag effect on the transverse colon and abdominal adhesion may occur when omentum was separated. Nonsurgical procedures, such as percutaneous aspiration, have also been proposed for simple cysts. Percutaneous aspiration is traditionally followed by the injection of $95 \%$ ethanol under ultrasound guidance. However, intolerable pain coupled with an almost $100 \%$ level of relapse make this procedure highly contentious $(30,31)$. Also, with repeated interventions, patients reported very limited relief of their symptoms (32). In recent years, a refined surgical approach has been developed to apply ethanol sclerotherapy with laparoscopic deroofing (33), and has significantly reduced recurrence rate and major complications (34). However, ethanol is not feasible for patients with alcohol allergies and was also occasionally reported to cause liver function problems. Alternatively, lauromacrogol is a proper sclerosant with a mild anesthetic effect. The injected lauromacrogol is associated with a higher cure rate and a lower incidence of adverse reactions (35).

Therefore, the laparoscopic lauromacrogol sclerotherapy refined surgical approach was selected by the authors as an alternative to laparoscopic omentoplasty. This analysis retrospectively reviewed data from 49 patients admitted to the Department of Hepatobiliary Surgery of the Affiliated Hospital of Jiangnan University between October 2015 and June 2020. All patients had simple hepatic cysts located in the IVa, VII, and VIII segments. Both surgical approaches achieved good therapeutic effect, although laparoscopic lauromacrogol sclerotherapy showed a greater reduction in cyst volume compared to laparoscopic omentoplasty. This might be because the omentum can drain and absorb cyst fluid. For laparoscopic omentoplasty, the cyst cavity may be occupied by the omentum tissue, as is shown in Figure 3, resulting in a comparatively larger average postoperative cyst volume. In contrast, laparoscopic lauromacrogol sclerotherapy applies sclerosing agent to the cyst wall without cyst fluid or the omentum flap, and thus, the cyst volume can be reduced significantly following surgery. As for quality of life, both groups reported a generally satisfactory health status. Although, we observed slightly higher scores from patients who received laparoscopic lauromacrogol sclerotherapy reporting their general health status and current health in comparison to 1-year prior.

In summary, we confirm that laparoscopic lauromacrogol sclerotherapy is an effective alternative to laparoscopic omentoplasty. With regards to surgical technique, we propose three important recommendations for surgeons. First, the incision of the cyst wall should be small to effectively prevent overflow of the sclerosing foam, which can potentially lead to postoperative pain. Second, the position of patients needs to be adjusted when injecting lauromacrogol so that the capsule wall covered with liver tissue is always in contact with sclerosing agent. Finally, following sclerotherapy, the cysts should be flushed with saline to prevent lauromacrogol foam residue.

Due to a relatively small sample size, this research is subject to some limitations. Therefore, a longer period of study, a larger sample size and some non-parametric estimations, for example, bootstrap can be applied in the future research. In addition, there are some uncertainties in retrospective research. The uncertainties can partially be solved by keeping complete medical records of patients and their follow up documents.

\section{Conclusions}

This study confirms that laparoscopic lauromacrogol sclerotherapy can be performed safely and effectively for simple hepatic cysts in the difficult IVa, VII, and VIII hepatic segments. Using this approach, we found no recurrences, a significant reduction in cyst volume, and a satisfactory quality of life status in patients at the 3-8 months follow-up stage. We propose that the laparoscopic lauromacrogol sclerotherapy is worth promoting as an effective alternative to laparoscopic omentoplasty, especially for patients that have the following characteristics: (I) cysts that are located in IVa, VII, and VIII segments; (II) the lack of omentum flap to cover the extensively fenestrated hepatic surface; (III) a history of abdominal surgery or severe omentum adhesion.

\section{Acknowledgments}

Funding: This research was funded by the Wuxi Health Commission project; grant number Q201768.

\section{Footnote}

Reporting Checklist: The authors have completed the STROBE reporting checklist. Available at http://dx.doi. org/10.21037/apm-20-1723

Data sharing statement: Available at http://dx.doi. 
org/10.21037/apm-20-1723

Conflicts of Interest: All authors have completed the ICMJE uniform disclosure form (available at http://dx.doi. org/10.21037/apm-20-1723). The authors have no conflicts of interest to declare.

Ethical Statement: The authors are accountable for all aspects of the work in ensuring that questions related to the accuracy or integrity of any part of the work are appropriately investigated and resolved. The study was conducted in accordance with the Declaration of Helsinki (as revised in 2013). The study was approved by the Medical Ethics Committee of the Affiliated Hospital of Jiangnan University (No. LS2015041) and written informed consent was obtained from all patients.

Open Access Statement: This is an Open Access article distributed in accordance with the Creative Commons Attribution-NonCommercial-NoDerivs 4.0 International License (CC BY-NC-ND 4.0), which permits the noncommercial replication and distribution of the article with the strict proviso that no changes or edits are made and the original work is properly cited (including links to both the formal publication through the relevant DOI and the license). See: https://creativecommons.org/licenses/by-nc-nd/4.0/.

\section{References}

1. Gall TMH, Oniscu GC, Madhavan K, et al. Surgical management and longterm follow-up of non-parasitic hepatic cysts. HPB 2009;11:235-41.

2. Moorthy K, Mihssin N, Houghton PW. The management of simple hepatic cysts: sclerotherapy or laparoscopic fenestration. Ann R Coll Surg Engl 2001;83:409-14.

3. Leung TK, Lee CM, Chen HC. Fatal thrombotic complications of hepatic cystic compression of the inferior vena: A case report. World J Gastroenterol 2005;11:1728-9.

4. Day RJ, Sanchirico PJ, Pfeiffer DC. Giant hepatic cyst as a cause of gastric outlet obstruction. Radiol Case Rep 2019;14:1088-92.

5. Lee DM, Kwon OS, Choi YI, et al. [Spontaneously Resolving of Huge Simple Hepatic Cyst]. Korean J Gastroenterol 2018;72:86-9.

6. Faulds JM, Scudamore CH. Technical report of a novel surgical technique: laparoscopic cyst fenestration and falciform ligament pedicle graft for treatment of symptomatic simple hepatic cysts. J Laparoendosc Adv Surg Tech A 2010;20:857-61.

7. Akhan O, Islim F, Balci S, et al. Percutaneous Treatment of Simple Hepatic Cysts: The Long-Term Results of PAIR and Catheterization Techniques as Single-Session Procedures. Cardiovasc Intervent Radiol 2016;39:902-8.

8. Mazza OMMD, Fernandez DLMD, Pekolj JMDPF, et al. Management of Nonparasitic Hepatic Cysts. J Am Coll Surg 2009;209:733-9.

9. Vardakostas D, Damaskos C, Garmpis N, et al. Minimally invasive management of hepatic cysts: indications and complications. Eur Rev Med Pharmacol Sci 2018;22:1387-96.

10. Macedo FI. Current management of noninfectious hepatic cystic lesions: A review of the literature. World J Hepatol 2013;5:462-9.

11. Antonacci N, Ricci C, Taffurelli G, et al. Systematic review of laparoscopic versus open surgery in the treatment of non-parasitic liver cysts. Updates Surg 2014;66:231-8.

12. Killeen S, Mannion M, Devaney A, et al. Omentoplasty to assist perineal defect closure following laparoscopic abdominoperineal resection. Colorectal Dis 2013;15:e623-6.

13. Gomez IGC, López-Andújar R, Belda Ibáñez T, et al. Review of the treatment of liver hydatid cysts. World J Gastroenterol 2015;21:124-31.

14. Tikkakoski T, Mäkelä JT, Leinonen S, et al. Treatment of Symptomatic Congenital Hepatic Cysts with Single-Session Percutaneous Drainage and Ethanol Sclerosis: Technique and Outcome. J Vasc Interv Radiol 1996;7:235-9.

15. Wijnands TF, Gortjes AP, Gevers TJ, et al. Evaluation of Efficacy and Safety of Percutaneous Aspiration Sclerotherapy of Simple Hepatic Cysts, a Systematic Review. J Hepatol 2015;64:S702-3.

16. Debs T, Kassir R, Reccia I, et al. Technical challenges in treating recurrent non-parasitic hepatic cysts. Int J Surg 2016;25:44-8.

17. Belghiti J. How could we image the future in hepatic surgery. Hepatobiliary Surg Nutr 2016;5:279.

18. Lin TY, Chen CC, Wang SM. Treatment of non-parasitic cystic disease of the liver: a new approach to therapy with polycystic liver. Ann Surg 1968;168:921.

19. Martin IJ, McKinley AJ, Currie EJ, et al. Tailoring the Management of Nonparasitic Liver Cysts. Ann Surg 1998;228:167-72.

20. Jia C, Li H, Wen N, et al. Laparoscopic liver resection: a review of current indications and surgical techniques. 
Hepatobiliary Surg Nutr 2018;7:277-88.

21. Tan YM, Chung A, Mack P, et al. Role of fenestration and resection for symptomatic solitary liver cysts. ANZ J Surg 2005;75:577-80

22. Zacherl J, Scheuba C, Imhof M, et al. Long-term results after laparoscopic unroofing of solitary symptomatic congenital liver cysts. Surg Endosc 2000;14:59-62.

23. Maruyama Y, Okuda K, Ogata T, et al. Perioperative challenges and surgical treatment of large simple, and infectious liver cyst - a 12-year experience. PLoS One 2013;8:e76537.

24. Petri A, Höhn J, Makula É, et al. Experience with different methods of treatment of nonparasitic liver cysts. Langenbecks Arch Surg 2002;387:229-33.

25. Kwon AH, Matsui Y, Inui H, et al. Laparoscopic treatment using an argon beam coagulator for nonparasitic liver cysts. Am J Surg 2003;185:273-7.

26. Ardito F, Bianco G, Vellone M, et al. Long-term outcome after laparoscopic fenestration of simple liver cysts. Surg Endosc 2013;27:4670-4.

27. Weber T, Sendt W, Scheele J. Laparoscopic Unroofing of Nonparasitic Liver Cysts Within Segments VII and VIII:

Technical Considerations. J Laparoendosc Adv Surg Tech A 2004;14:37-42.

28. Palanivelu C, Jani K, Malladi V. Laparoscopic Management of Benign Nonparasitic Hepatic Cysts: A Prospective Nonrandomized Study. Southern Med J

Cite this article as: $\mathrm{Xu} \mathrm{S,} \mathrm{Rao} \mathrm{M,} \mathrm{Pu} \mathrm{Y,} \mathrm{Zhou} \mathrm{J,} \mathrm{Zhang} \mathrm{Y.} \mathrm{The}$ efficacy of laparoscopic lauromacrogol sclerotherapy in the treatment of simple hepatic cysts located in posterior segments: a refined surgical approach. Ann Palliat Med 2020;9(5):3462-3471. doi: 10.21037/apm-20-1723
2006;99:1063-7.

29. Konstadoulakis MM, Gomatos IP, Albanopoulos K, et al. Laparoscopic fenestration for the treatment of patients with severe adult polycystic liver disease. Am J Surg 2005;189:71-5.

30. Regev A, Reddy KR, Berho M, et al. Large cystic lesions of the liver in adults: a 15-year experience in a tertiary center. J Am Coll Surg 2001;193:36.

31. Agarwal MM, Hemal AK. Surgical Management of Renal Cystic Disease. Curr Urol Rep 2011;12:3-10.

32. Neri V, Ambrosi A, Fersini A, et al. Laparoscopic treatment of biliary hepatic cysts: short- and medium-term results. HPB 2006;8:306-10.

33. Efesoy O, Tek M, Bozlu M, et al. Comparison of single-session aspiration and ethanol sclerotherapy with laparoscopic de-roofing in the management of symptomatic simple renal cysts. Turk J Urol 2015;41:14-9.

34. De Cicco Nardone A, Carfagna P, De Cicco Nardone C, et al. Laparoscopic Ethanol Sclerotherapy for Ovarian Endometriomas: Preliminary Results. J Minim Invasive Gynecol 2020;S1553-4650(20)30224-7.

35. Linghu E, Du C, Chai N, et al. A prospective study on the safety and effectiveness of using lauromacrogol for ablation of pancreatic cystic neoplasms with the aid of EUS. Gastrointest Endosc 2017;86:872-80.

(English Language Editor: A. Kassem) 